\title{
Iron mediates endothelial cell damage and blood-brain barrier opening in the hippocampus after transient forebrain ischemia in rats
}

\author{
Sun Mi Won ${ }^{1,4 *}$, Jin Hwan Lee $e^{1,4,5 *}$, \\ Ui Jin Park ${ }^{1,4,5}$, Jina Gwag ${ }^{1}$, \\ Byoung Joo Gwag ${ }^{1,4,5,6}$ and Yong Beom Lee ${ }^{1,2,3,6}$ \\ ${ }^{1}$ Neuroscience Graduate Program \\ ${ }^{2}$ Brain Disease Research Center \\ ${ }^{3}$ Institute for Medical Science \\ ${ }^{4}$ Department of Pharmacology \\ Ajou University School of Medicine \\ Suwon 443-721, Korea \\ ${ }^{5}$ Neurotech Pharmaceuticals Co. Ltd., \\ Suwon 443-721, Korea \\ ${ }^{6}$ Corresponding authors: Tel, 82-31-219-4221; \\ Fax, 82-31-219-5069; E-mail, bjgwag5001@ gmail.com (B.J.G.); \\ Tel, 82-31-219-4558; Fax, 82-31-219-4530; \\ E-mail, yblee@ ajou.ac.kr (Y.B.L.)
}

*These authors contributed equally to this work.

DOI 10.3858/emm.2011.43.2.020

Accepted 28 January 2011

Available Online 31 January 2011

Abbreviations: BBB, blood-brain barrier; CEC, cerebral endothelial cell; DFO, deferoxamine; MMP-2, matrix metalloproteinase-2; TFI, transient forebrain ischemia; TJ, tight junction; ZO, zonula occludens

\begin{abstract}
Blood cells are transported into the brain and are thought to participate in neurodegenerative processes following hypoxic ischemic injury. We examined the possibility that transient forebrain ischemia (TFI) causes the blood-brain barrier (BBB) to become permeable to blood cells, possibly via dysfunction and degeneration of endothelial cells in rats. Extravasation of Evans blue and immunoglobulin G (IgG) was observed in the hippocampal CA1-2 areas within $8 \mathrm{~h}$ after TFI, and peaked at $48 \mathrm{~h}$. This extravasation was accompanied by loss of tight junction proteins, occludin, and zonula occludens-1, and degeneration of endothelial cells in the CA1-2 areas. Iron overload and mitochondrial free radical production were evident in the microvessel endothelium of the hippocampus before endothelial cell damage occurred. Administration of deferoxamine (DFO), an iron chelator, or Neu2000, an antioxidant,
\end{abstract}

blocked free radical production and endothelial cell degeneration. Our findings suggest that iron overload and iron-mediated free radical production cause loss of tight junction proteins and degeneration of endothelial cells, opening of the BBB after TFI.

Keywords: blood-brain barrier; deferoxamine; free radical; Neu2000; transient forebrain ischemia; iron

\section{Introduction}

The blood-brain barrier (BBB) plays a critical role in the maintenance of ionic homeostasis within the CNS, and in communication between the CNS and peripheral tissues (Hawkins and Davis, 2005; Abbott et al., 2010). Deregulation of BBB integrity has been well documented following hypoxic ischemic brain injury (Kuroiwa et al., 1988; Belayev et al., 1996; del Zoppo and Mabuchi, 2003), and has also been reported to feature in other neurological diseases including neurodegenerative conditions (Zlokovic, 2008), autoimmune disorders (Minagar and Alexander, 2003), and post-infection with viruses, bacteria, or other microorganisms (Kim, 2008). BBB damage results in extravasation of blood cells, extracellular fluid, and macromolecules into the CNS parenchyma. Cytotoxic molecules including nitric oxide, proteases, and inflammatory cytokines are then produced within and released from the infiltrating leukocytes, contributing to neuronal cell injury (Rezai-Zadeh et al., 2009).

Hypoxic ischemia induces expression of inflammatory cytokines including TNF- $\alpha$, IL-1 $\beta$, and IL-6; these molecules stimulate the production and release of chemokines, which are chemoattractant proteins known to mediate recruitment and infiltration of leukocytes (Huang et al., 2006). In knockout chemoattractant protein-1 (MCP-1) mice, MCP-1 is a potent chemoattractant and activator of monocytes/ macrophages, leukocyte infiltration and brain edema following occlusion of the middle cerebral artery are significantly reduced (Dimitrijevic et al., 2007). Recruited neutrophils and mononuclear phagocytes induce upregulation of iNOS, matrix metalloproteinase-2 (MMP-2) and -9, and VEGF; these peptides cause disruption of BBB integrity via degradation of vascular extracellular matrix and tight junctions (Petty 
and Lo, 2002).

Excess free radicals are produced in brain areas subject to marked degeneration of neurons and glia following hypoxic ischemic injury (Won et al., 2002; Sugawara and Chan, 2003; Choi et al., 2007). Free radicals are known to negatively impact intercellular junction functions by modulating adherence of tight junction (TJ) proteins, and reorganization of the actin filament network within vascular endothelial cells (Lum and Roebuck, 2001; Usatyuk et al., 2006). Pharmacological treatments seeking to remove free radicals prevent BBB breakdown and neuronal death following focal cerebral ischemia (Kim et al., 2001; Ding-Zhou et al., 2002). Iron ions, serving as transition metal molecules catalyzing hydroxyl radical production via the Fenton reaction and the Haber-Weiss cycle, accumulate in neurons, astrocytes, and microglia in hippocampal CA1 areas, after occurrence of transient forebrain ischemia (TFI), and cause neuronal death and gliosis (Kondo et al., 1995; Wang et al., 2008). In the present study, we explored the possibility that iron-mediated free radical production mediates endothelial cell damage and BBB opening in the hippocampal CA1-2 regions, following TFI.

\section{Results}

\section{Temporal and spatial patterns of BBB disruption following TFI}

We examined the temporal pattern of BBB permeability in the hippocampus after TFI. Western blotting showed that the levels of both the light (25 $\mathrm{kDa})$ and heavy $(50 \mathrm{kDa})$ chains of serum $\mathrm{lgG}$ increased in the hippocampus, commencing $8 \mathrm{~h}$ after TFI (Figure 1A and B). IgG levels increased further (by up to 6 -fold) over $48 \mathrm{~h}$, and remained elevated, by approximately 2.5 -fold, $72 \mathrm{~h}$ after TFI. Double-labeling with both Evans blue dye (EBD)-albumin and an antibody against endothelial barrier antigen (EBA) revealed that extravasation of EBD-albumin was evident in the microvasculature of the CA1-2 pyramidal cell layer $48 \mathrm{~h}$ after reperfusion, compared to data from the shamoperated control (Figure 1C), suggesting that BBB permeability increased primarily in CA1-2 areas after TFI. At this time point, EBD-albumin was not detectable in other hippocampal areas, including the CA3 region and the dentate gyrus. This implies that TFI selectively disrupts the BBB in the hippocampal CA1-2 areas that are particularly vulnerable to ischemic injury.
A
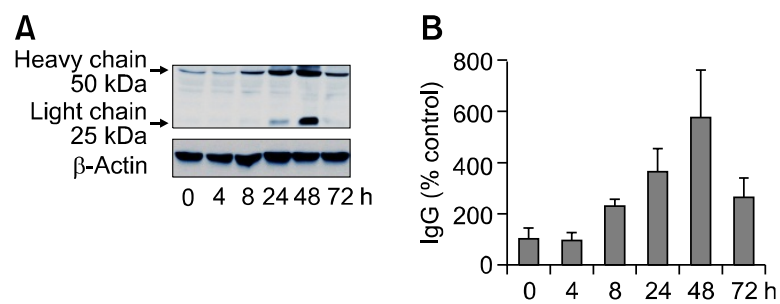

C
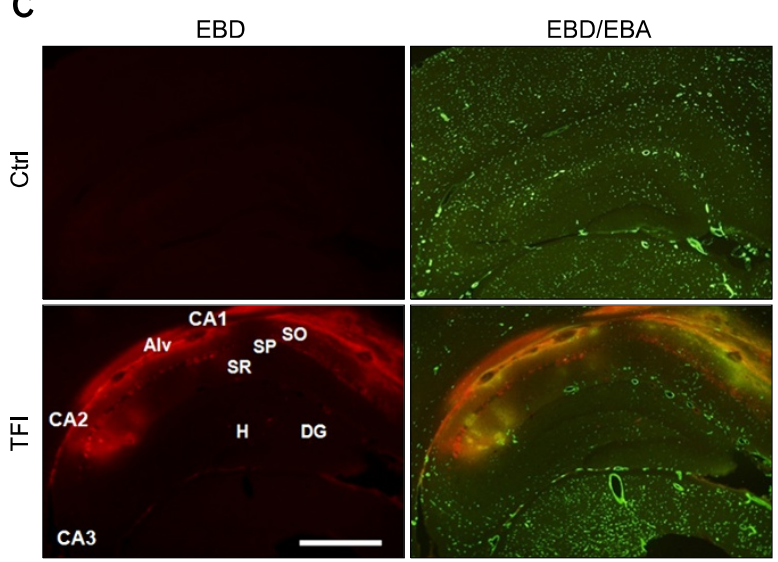

Figure 1. Temporal and spatial patterns of BBB opening in the hippocampus following TFI. (A, B) Western blot analysis of IgG showing increase in the light and heavy chains of $\mathrm{IgG}$ after sham operation (Ctrl) or TFI. Levels of IgG were measured at indicated points of time after TFI, normalized to those of $\beta$-actin, and compared to control ( $n=4-5$ rats per group). (C) Fluorescent photomicrographs showing EBD-albumin extravasation and cerebral endothelium stained with EBA, in the hippocampal formation, $48 \mathrm{~h}$ after Ctrl or TFI. Note the dramatic increase in EBD extravasation in the endothelium (yellow), primarily in the alveus (Alv), stratum oriens (SO), and stratum pyramidale (SP) of the CA1-2 areas. Abbreviations: $\mathrm{DG}$, dentate gyrus; $\mathrm{H}$, hilus, $\mathrm{SR}$, stratum radiatum. Scale bar: $1.0 \mathrm{~mm}$.

\section{Loss of occludin and ZO-1 proteins, and endothelial cell degeneration in the CA1-2 regions, after TFI}

Reduced expression of TJ proteins including occludin and ZO-1 disrupts TJ and increases BBB permeability (Hirase et al., 1997). Western blotting showed that the levels of ZO-1 and occludin were significantly reduced within 8 and 24 h after TFI, respectively, and loss of the TJ proteins were still evident $48 \mathrm{~h}$ after TFI (Figure 2A). Immunohistochemistry showed that occludin was normally expressed in cerebral endothelial cells (CECs), and its expression was markedly reduced in the CECs of CA1-2 areas $48 \mathrm{~h}$ after TFI (Figure 2B). Moreover, the terminal deoxynucleotidyl transferase dUTP nick-end labeling (TUNEL) assay, and immunostaining with anti-EBA antibody, performed on adjacent brain sections, showed that TUNEL-positive signals were widely apparent in the condensed and fragmented EBA-positive CECs of CA1-2 areas $48 \mathrm{~h}$ after TFI (Figure $2 \mathrm{C}$ ), but not in the CA3 and dentate gyrus (data not shown). 
A
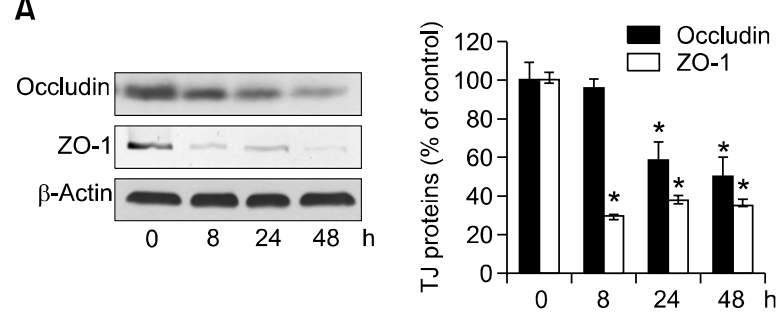

B
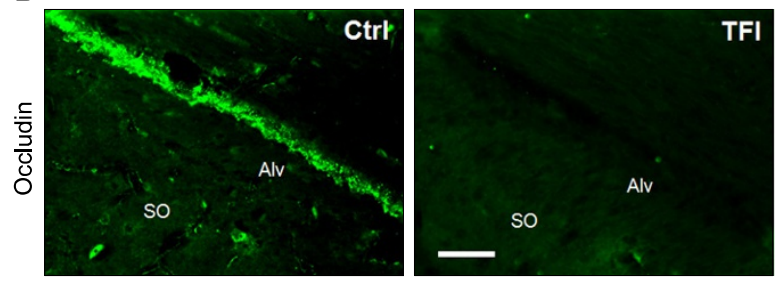

C
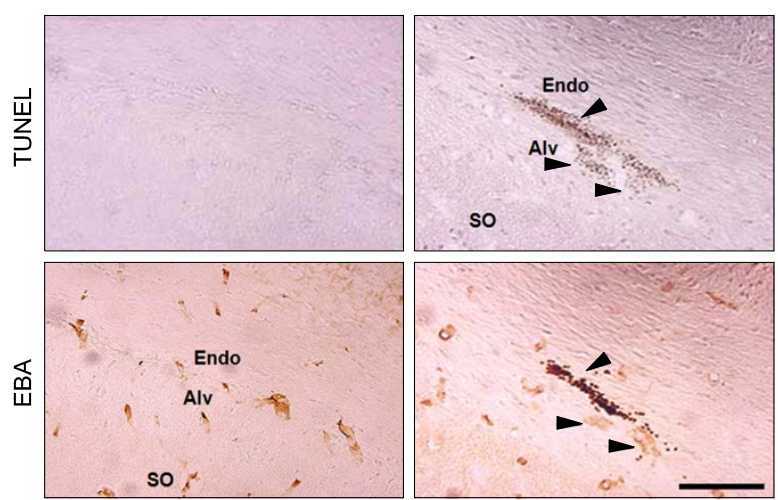

Figure 2. Loss of occludin and ZO-1 and degeneration of CECs in the hippocampus after TFI. (A) Western blot analysis of occludin and ZO-1. Levels of occludin and ZO-1 were measured 8, 24, and $48 \mathrm{~h}$ after TFI, normalized to those of $\beta$-actin, and compared to control $(n=3-4$ rats per group). ${ }^{*}, P<0.05$ compared to control. (B) Fluorescence photomicrographs of the hippocampal CA1 area immunolabeled with occludin $48 \mathrm{~h}$ after control (Ctrl) or TFI. Scale bar, $50 \mu \mathrm{m}$. (C) Brightfield photomicrographs of adjacent hippocampal CA1 sections showing DNA fragmentation after TUNEL staining (top panel) and endothelial cells after immunolabeling with EBA (bottom panel) $48 \mathrm{~h}$ after Ctrl or TFI. Arrowheads show degeneration of endothelial cells. Abbreviations: Alv, alveus; Endo, endothelium; SO, stratum oriens. Scale bar, $100 \mu \mathrm{m}$.

\section{Region-specific iron overload-mediated free radical production in CECs after TFI}

We have previously shown that iron overload is evident early in CA1 pyramidal neurons after TFI, in turn mediating free radical production and delayed neuronal degeneration (Wang et al., 2008; Park et al., 2010). In this context, iron overload likely contributes to degeneration of CECs, and opening of the BBB. Perls iron staining revealed that iron overload was detectable in the CA1-2 areas within $0.5 \mathrm{~h}$ of TFI, and that the effect lasted for $2 \mathrm{~h}$ (Figure 3A). Perls staining and EBA immuno-
A

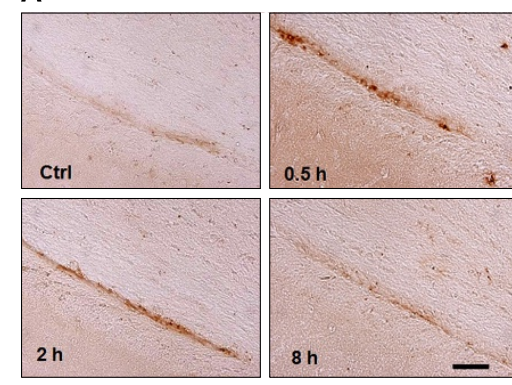

B

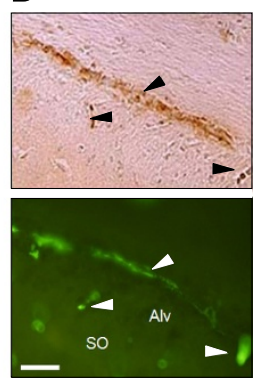

C

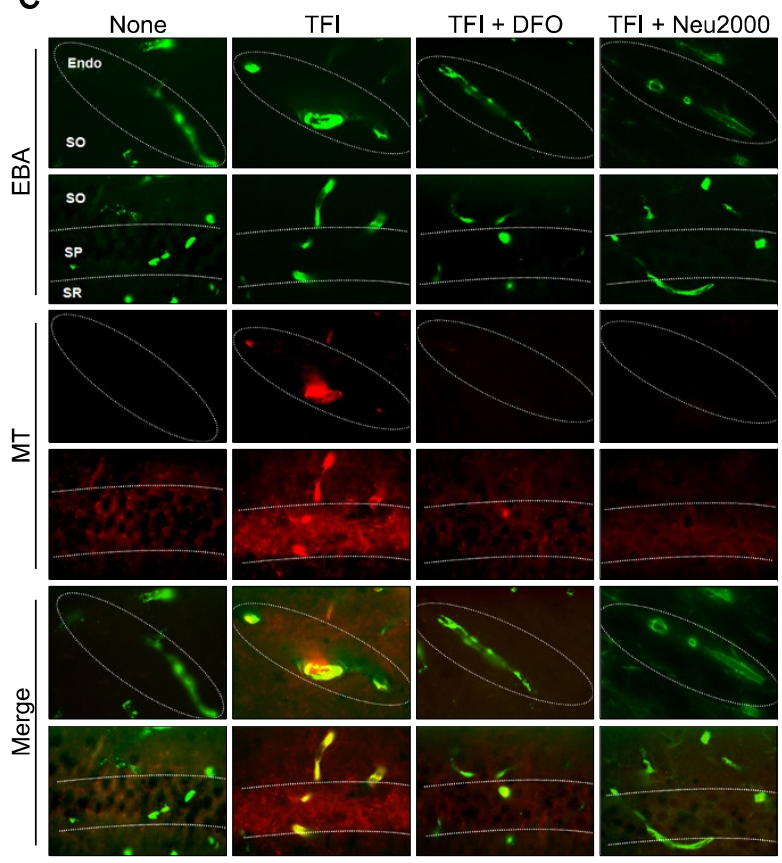

Figure 3. Iron overload and free radical production in the CECs after TFI. (A) Brightfield photomicrographs of the hippocampal CA1 area after Perls iron staining following sham operation (Ctrl) or $0.5,2$, and $8 \mathrm{~h}$ following TFI. Scale bar, $50 \mu \mathrm{m}$. (B) Brightfield and fluorescence photomicrographs of adjacent hippocampal CA1 sections after Perls staining (top panel) or immunolabeling with EBA (bottom panel) $0.5 \mathrm{~h}$ after TFI. Arrowheads indicate iron overload in endothelial cells. Scale bar, $50 \mu \mathrm{m}$. (C) Superimposed images of fluorescence photomicrographs of hippocampal sections labeled with EBA and MitoTracker Red CM-H2XROs (MT, red), a mitochondrial free radical probe, $2 \mathrm{~h}$ after Ctrl or TFI, alone or with administration of DFO (200 mg/kg, s.c.) or Neu2000 $(50 \mathrm{mg} / \mathrm{kg}$; i.p.) immediately after reperfusion. Abbreviations: Endo, endothelium; SO, stratum oriens; SP, stratum pyramidale; SR, stratum radiatum. Scale bar, $50 \mu \mathrm{m}$.

histochemistry of adjacent brain sections showed that iron content was increased, after TFI, in CECs located in the alveus and stratum oriens of the CA1-2 areas (Figure 3B), but not in cells of the CA3 and dentate gyrus (data not shown). The levels of mitochondrial free radicals (MFRs), analyzed by the oxidized fluorescent products of Mitotracker Red $\mathrm{CM}-\mathrm{H}_{2} \mathrm{XRos}$, rose in CECs of the 

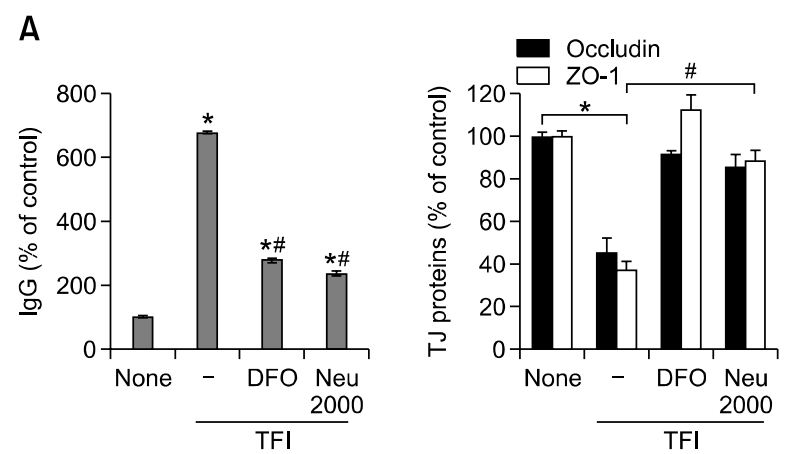

B

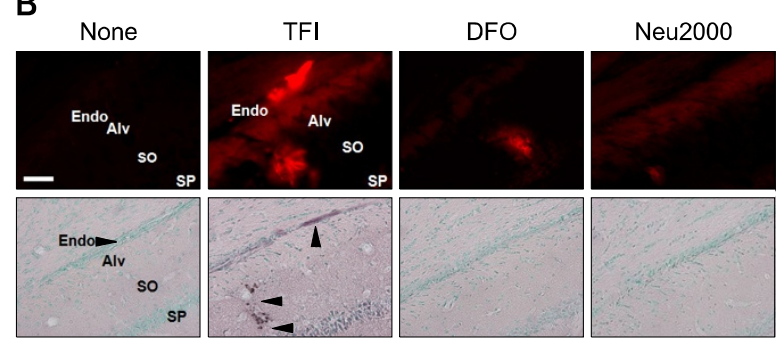

Figure 4. DFO and Neu2000 protect cerebral endothelial cells against TFI. (A) Western blot analysis of IgG and TJ proteins $48 \mathrm{~h}$ after Ctrl or $\mathrm{TFI}$, alone or with administration of DFO $(200 \mathrm{mg} / \mathrm{kg}$, s.c.) or Neu2000 (50 mg/kg; i.p.) immediately after reperfusion ( $n=3-4$ rats per group). ${ }^{*} P$ $<0.05$ compared to Ctrl; ${ }^{\sharp} P<0.05$ compared to TFI. (B) Fluorescent and brightfield photomicrographs of hippocampal CA1 sections labeled with EBD-albumin (red, top panel) or TUNEL and methyl green, a chromatin staining dye (bottom panel) $48 \mathrm{~h}$ after Ctrl or TFI, alone or with administration of DFO (200 mg/kg, s.c.) or Neu2000 (50 mg/kg; i.p.) immediately after reperfusion. Arrowheads marked the endothelium and CECs of CA1-2 areas. Abbreviations: Alv, alveus; Endo, endothelium; SO, stratum oriens; SP, stratum pyramidale. Scale bar, $50 \mu \mathrm{m}$.

CA1-2 areas $2 \mathrm{~h}$ after TFI (Figure 3C). Administration of deferoxamine mesylate (DFO) $(200$ $\mathrm{mg} / \mathrm{kg}$; s.c.), a selective iron chelator, or Neu2000 (50 $\mathrm{mg} / \mathrm{kg}$, i.p.), a potent spin trapping molecule derived from aspirin and sulfasalazine (Gwag et al., 2007) immediately after reperfusion, almost completely blocked TFI-induced MFRs production $2 \mathrm{~h}$ after TFI (Figure $3 \mathrm{C}$ ).

\section{BBB disruption in CA1-2 regions via iron overload-mediated free radical production}

Next, we examined whether iron-mediated free radicals caused BBB disruption after TFI. Administration of either DFO $(200 \mathrm{mg} / \mathrm{kg}$, s.c.) or Neu2000 (50 $\mathrm{mg} / \mathrm{kg}$, i.p.) attenuated levels of serum $\mathrm{lgG}$, the loss of occludin and ZO-1 (Figure 4A), and the extravasation of the EBD-albumin (Figure 4B), $48 \mathrm{~h}$ after TFI. We also observed that administration of DFO or Neu2000 almost completely blocked TUNEL-positive signaling from the endothelium of CA1-2 areas (Figure 4B). These results indicate that iron-mediated free radical production causes
BBB disruption via loss of TJ proteins, and death of CECs, after TFI.

\section{Discussion}

We demonstrate that TFI causes BBB opening primarily in the hippocampal CA1-2 areas. The BBB opening is attributable to free radical-mediated loss of TJ proteins, occludin and ZO-1, and degeneration of CECs. This effect is attenuated by DFO, an iron chelator, or Neu2000, an antioxidant that near completely blocks free radical generation after TFI.

Increased BBB permeability has been reported in brains subjected to TFI in normal and spontaneously hypertensive rats (Dobbin et al., 1989; Preston et al., 1993; Abraham et al., 2002). Radiotracer studies using $\left[{ }^{3} \mathrm{H}\right]$-sucrose or $\left[{ }^{14} \mathrm{C}\right]$-amino isobutyric acid have revealed transient BBB opening in the neocortex, striatum, and hippocampus, after TFI (Dobbin et al., 1989; Preston et al., 1993). Levels of $\left[{ }^{3} \mathrm{H}\right]$-sucrose were increased in the hippocampus $6 \mathrm{~h}$ after 10-min 2-vessel occlusion (VO) ischemia in normal rats but gradual increase over $24 \mathrm{~h}$ after 25-min TFI or 10-min TFI combined with hyperthermia (Preston et al., 1993), and EBDalbumin levels were increased in the hippocampus between 6 and $24 \mathrm{~h}$ following 10-min 2-VO ischemia in stroke-prone spontaneously hypertensive rats (Abraham et al., 2002). We observed increases in IgG levels in the hippocampus, over $72 \mathrm{~h}$ after 4 -VO model of TFI (10-min). This suggests that the temporal patterns of BBB permeability depend upon severity of the ischemic injury. Histochemical assessment of EBD-albumin revealed that BBB permeability was increased primarily in the hippocampal CA1-2 areas before occurrence of neuronal death in the CA1. This implies that BBB opening and the resulting infiltration of blood cells may contribute to delayed degeneration of CA1 neurons after TFI.

TJ proteins, including occludin and ZO-1, play a role in regulation of the paracellular permeability barrier via fusion of the endothelial plasma membrane (Sandoval and Witt, 2008). The C-terminal domain of occludin is cross-linked with actin in a process mediated by cytoplasmic ZO proteins (Furuse et al., 1994). Thus, loss of the TJ proteins causes increase in paracellular permeability. Defective occludin and ZO-1 expression was reported in endothelial cells and correlated with BBB opening after induction of embolic cerebral ischemia (Date et al., 2006) and transient focal ischemia (Asahi et al., 2001). We observed that ZO-1 and occludin levels were significantly reduced within 8 and $24 \mathrm{~h}$ 
after TFI, respectively, indicating that BBB opening commences with loss of TJ proteins. Endothelial cell death, another cause of BBB opening, has been reported following TFI or oxygen-glucose deprivation (Kitagawa et al., 1992; Hayashi et al., 2001; Yin et al., 2010). In the present study, TUNEL staining revealed degeneration of CECs selectively in the alveus and stratum oriens of the CA1-2 regions $48 \mathrm{~h}$ after TFI. This implies that TJ protein loss and CEC degeneration contribute to TFI-induced BBB opening.

Iron-mediated free radical production mediates neuronal death and gliosis in the CA1 area after TFI (Kondo et al., 1995; Wang et al., 2008). Recent studies have shown that oxidative stress is a prominent feature of cerebral endothelial injury after cerebral ischemia (Maier et al., 2006; Jin et al., 2008). The lipophilic iron chelator dipyridyl was shown to prevent degeneration of CECs following photothrombotic ischemia (Methy et al., 2008). Thus, it is conceivable to reason that iron may mediate TJ protein loss and CEC degeneration after TFI. We observed that TFI induced iron overload and free radical production in the endothelium of CA1-2 areas within 0.5 and $2 \mathrm{~h}$, respectively. Administration of DFO blocked TFIinduced iron overload and free radical production. Loss of occludin and ZO-1, degeneration of CECs, and BBB opening were prevented by administration of DFO and Neu2000, a potent free radical scavenger, suggesting a causative role of ironmediated free radical production in endothelial cell damage and BBB opening after TFI. It remains to be delineated how iron is overloaded selectively in the endothelium of CA1-2 areas after TFI.

Brain endothelial cells contain relatively high amounts of ferritin, and hence serve as active reservoirs of iron (Burdo et al., 2004). Ferritin acts as an iron donor when exposed to metabolic conditions associated with ischemia that causes low oxygen tension, high levels of the superoxide anion, and acidosis (Bralet et al., 1992; Paul, 2000). Iron that is overloaded in CECs after TFI is likely released from ferritin. Excessive free iron then triggers free radical production by virtue of the Fenton reaction and the Haber-Weiss cycle. Iron-mediated free radical production significantly contributes to BBB opening as shown by sensitivity to DFO or Neu2000.

Our results support the hypothesis that ironmediated BBB disruption in the CA1-2 areas contributes to delayed neuronal cell death in the CA1 following TFI, for the time course of BBB opening is similar to the kinetics of free radical generation in CA1 neurons; free radical levels are highest 8 and $48 \mathrm{~h}$ after TFI (Wang et al., 2008;
Park et al., 2010). Iron overload was observed in the endothelium within $30 \mathrm{~min}$ after TFI, which resulted in delayed iron overload and free radical production in the CA1 neurons (Park et al., 2010). Furthermore, DFO prevents BBB opening and delayed degeneration of the CA1 neurons. Thus, CA1-specific iron accumulation in the endothelium may be a critical trigger inducing a cascade of events resulting in a region-specific BBB permeability increase, and neuronal damage in the early phase of forebrain ischemia.

\section{Methods}

\section{Animals}

Male Sprague-Dawley rats (Orient Bio Inc., Seongnam, Korea) weighing $250-300 \mathrm{~g}$, were used in all experiments involving TFI. All experimental procedures were conducted under the approval of the Institutional Animal Care and Use Committee of Ajou University School of Medicine.

\section{Transient forebrain ischemia}

TFI was induced by four-vessel occlusion, employing minor modifications of a method that has been previously described (Pulsinelli and Buchan, 1988). Briefly, rats were anesthetized by intraperitoneal (i.p.) injection of $400 \mathrm{mg} / \mathrm{kg}$ chloral hydrate (Fluka, Buchs, Switzerland), and the bilateral vertebral arteries were exposed and permanently electrocauterized. Both common carotid arteries (CCAs) were rendered accessible for subsequent occlusion. After $24 \mathrm{~h}$, fully-awake rats were restrained, and the CCAs were bilaterally occluded, using aneurysm clips, for $10 \mathrm{~min}$. Signs including an unresponsive loss of the righting reflex and catatonic posture were considered indicative of TFI development. Arteries were visually inspected to ensure adequate reflow after release of carotid artery clips. Body temperature was maintained at $37.5^{\circ} \mathrm{C}$, using heating pads, until animals recovered from surgery and/or ischemia. Animals were fasted for 1 day before induction of ischemia, and were allowed free access to food and water thereafter. Sham-operated animals were treated in a manner identical to that described above, but the vertebral and common carotid arteries were not occluded.

\section{Drug treatment}

Neu2000 (Neurotech Pharmaceuticals Co., Ltd, Suwon, Korea) was injected immediately (i.p.) after reperfusion, at a dose of $50 \mathrm{mg} / \mathrm{kg}$. Deferoxamine mesylate (Sigma Chemical Co., St. Louis, MO) was administered subcutaneously (s.c.), immediately after reperfusion, at $200 \mathrm{mg} /$ $\mathrm{kg}$.

\section{Tissue preparation and immunohistochemistry}

After animals were subjected to transcardiac perfusion using phosphate-buffered saline (PBS; $\mathrm{pH} 7.4$ ), and $3 \%$ paraformaldehyde; brains were fixed in $3 \%$ paraformal- 
dehyde and then immersed in 30\% sucrose for 3-4 days at $4^{\circ} \mathrm{C}$. Frozen brains were coronally sectioned, in the regions 3-4 $\mathrm{mm}$ posterior to the bregma, at a slice thickness of 25 $\mu \mathrm{m}$, using a Cryocut Microtome (Leica Microsystems, Wetzlar, Germany). After inactivation of endogenous peroxidase activity by hydrogen peroxide, the tissues were incubated in blocking solution (10\% normal goat serum in PBS) for 1 $\mathrm{h}$, followed by incubation with a primary antibody: mouse anti-endothelial barrier antigen (EBA) (1:1,000 dilution; Covance, Richmond, CA), or mouse anti-occludin (1:100; Zymed, San Francisco, CA). After $24 \mathrm{~h}$, sections were thoroughly washed with PBS, and incubated with fluorescein isothiocyanate-labeled horse anti-mouse antibody (1:200 dilution; Vector Laboratories, Burlingame, CA), for 2 $h$ at room temperature (RT).

\section{Iron histochemistry with Perls staining}

Iron was detected using 3,3'-diaminobenzidine tetrahydrochloride (DAB) enhancement of Prussian Blue staining (Hill and Switzer, 1984). In brief, fixed sections were incubated in $1 \%$ sodium borohydride (Sigma) for $30 \mathrm{~min}$, washed with PBS, and incubated in $30 \mu \mathrm{g} / \mathrm{ml}$ proteinase $\mathrm{K}$ (Sigma), with $0.1 \%$ Triton X-100, for 20 min. Sections were next incubated in Perls solution containing $2 \%$ potassium ferrocyanide and $2 \%$ hydrochloric acid, for $30 \mathrm{~min}$. The sections were washed with PBS and incubated in $0.05 \%$ DAB solution in $0.05 \mathrm{M}$ Tris- $\mathrm{HCl}$ ( $\mathrm{pH} 7.6$ ), containing $30 \%$ hydrogen peroxide $(2 \mu \mathrm{l} / \mathrm{ml})$, for $15 \mathrm{~min}$ in the dark.

\section{Extravasation of Evans blue dye (EBD)}

BBB permeability was investigated by monitoring extravasation of Evans blue dye (EBD). The red auto-fluorescence of EBD-albumin conjugate is evident in tissue sections examined by fluorescence microscopy (Matsuda et al., 1995). Animals were injected, through a dissected femoral vein, with $0.1 \mathrm{ml}$ of $3 \% \mathrm{EBD}$ (Sigma) in $0.9 \%$ saline. After $30 \mathrm{~min}$, rats were euthanized by transcardial perfusion using $200 \mathrm{ml}$ PBS. Extravasation of EBD-albumin was observed by fluorescence microscopy (excitation wavelength $557 \mathrm{~nm}$; emission wavelength $576 \mathrm{~nm}$ ).

\section{Western blotting}

Brain tissues from hippocampal region were homogenized in RIPA buffer (50 mM Tris-HCl, $150 \mathrm{mM} \mathrm{NaCl}, 1 \% \mathrm{NP}-40$, $1 \mathrm{mM}$ EDTA, $0.1 \%$ SDS and $0.01 \% \mathrm{NaN}_{3}, 1 \mathrm{mM} \mathrm{PMSF}, \mathrm{pH}$ 7.4). Proteins therein were resolved on $8-10 \%$ SDS-PAGE and transferred to nitrocellulose membranes, which were next incubated with appropriate primary antibodies. The antibodies were biotinylated anti-rat IgG (1:300 dilution; Vector Systems), or mouse anti-occludin (1:1,000 dilution; Zymed), or rabbit anti-ZO-1 (1:1,000 dilution; Zymed). Incubation with appropriate horseradish peroxidase (HRP)conjugated secondary antibodies (Cell Signaling, Beverly, MA) followed. After washing, electrochemiluminescence was assessed (Amersham, Buckinghamshire, UK), and image analysis (LAS1000; Fuji Photo Film Co., Ltd., Tokyo, Japan) afforded quantification of individual band intensity (Image Gauge 3.12; Fuji Photo Film Co., Ltd).

\section{Detection and analysis of mitochondrial free radicals}

Mitochondrial free radicals (MFRs, 3 rats for each group) were analyzed using MitoTracker Red $\mathrm{CM}-\mathrm{H}_{2} \mathrm{XR}_{\mathrm{os}}$ as previously described (Wang et al., 2008). In brief, animals were injected with $0.4 \mathrm{nmol}$ MitoTracker Red CM-H2XROS into the lateral ventricle through a Kopf stereotaxic apparatus $1 \mathrm{~d}$ prior to TFI. The brain sections were immunolabeled with an anti-EBA antibody to label endothelial cells. Mitochondrial free radicals were analyzed by monitoring the oxidized fluorescence product (excitation, $554 \mathrm{~nm}$; emission, $576 \mathrm{~nm}$ ) of MitoTracker Red CM$\mathrm{H}_{2}$ XRos.

\section{Statistical analysis}

All data were normalized to relevant control values (100\%) and results are expressed as mean \pm SEM. Statistical analysis was performed using one-way ANOVA, followed by Tukey test evaluation (SPSS, Chicago, IL).

\section{Acknowledgments}

This work was supported by grants from the 21C Frontier R\&D Program in Neuroscience from Ministry of Education, Science, and Technology, a National Drug Discovery Program from Ministry of Health and Welfare, the Brain Korea 21 project at Ajou University School of Medicine, and Neurotech Pharmaceuticals.

\section{References}

Abbott NJ, Patabendige AA, Dolman DE, Yusof SR, Begley DJ. Structure and function of the blood-brain barrier. Neurobiol Dis 2010;37:13-25

Abraham CS, Harada N, Deli MA, Niwa M. Transient forebrain ischemia increases the blood-brain barrier permeability for albumin in stroke-prone spontaneously hypertensive rats. Cell Mol Neurobiol 2002;22:455-62

Asahi M, Wang X, Mori T, Sumii T, Jung JC, Moskowitz MA, Fini ME, Lo EH. Effects of matrix metalloproteinase- 9 gene knock-out on the proteolysis of blood-brain barrier and white matter components after cerebral ischemia. J Neurosci 2001;21:7724-32

Belayev L, Busto R, Zhao W, Ginsberg MD. Quantitative evaluation of blood-brain barrier permeability following middle cerebral artery occlusion in rats. Brain Res 1996;739:88-96

Bralet J, Schreiber L, Bouvier C. Effect of acidosis and anoxia on iron delocalization from brain homogenates. Biochem Pharmacol 1992;43:979-83

Burdo JR, Simpson IA, Menzies S, Beard J, Connor JR. Regulation of the profile of iron-management proteins in brain microvasculature. J Cereb Blood Flow Metab 2004;24: 67-74

Choi YS, Cho KO, Kim EJ, Sung KW, Kim SY. Ischemic preconditioning in the rat hippocampus increases antioxidant activities but does not affect the level of hydroxyl radicals 
during subsequent severe ischemia. Exp Mol Med 2007;39: 556-63

Date I, Takagi N, Takagi K, Tanonaka K, Funakoshi H, Matsumoto K, Nakamura T, Takeo S. Hepatocyte growth factor attenuates cerebral ischemia-induced increase in permeability of the blood-brain barrier and decreases in expression of tight junctional proteins in cerebral vessels. Neurosci Lett 2006;407:141-5

del Zoppo GJ, Mabuchi T. Cerebral microvessel responses to focal ischemia. J Cereb Blood Flow Metab 2003;23:879-94

Dimitrijevic OB, Stamatovic SM, Keep RF, Andjelkovic AV. Absence of the chemokine receptor CCR2 protects against cerebral ischemia/reperfusion injury in mice. Stroke 2007;38:1345-53

Ding-Zhou L, Marchand-Verrecchia C, Croci N, Plotkine M, Margaill I. L-NAME reduces infarction, neurological deficit and blood-brain barrier disruption following cerebral ischemia in mice. Eur J Pharmacol 2002;457:137-46

Dobbin J, Crockard HA, Ross-Russell R. Transient bloodbrain barrier permeability following profound temporary global ischaemia: an experimental study using 14C-AIB. J Cereb Blood Flow Metab 1989;9:71-8

Furuse M, Itoh M, Hirase T, Nagafuchi A, Yonemura S, Tsukita S. Direct association of occludin with ZO-1 and its possible involvement in the localization of occludin at tight junctions. J Cell Biol 1994;127:1617-26

Gwag BJ, Lee YA, Ko SY, Lee MJ, Im DS, Yun BS, Lim HR, Park SM, Byun HY, Son SJ, Kwon HJ, Lee JY, Cho JY, Won SJ, Kim KW, Ahn YM, Moon HS, Lee HU, Yoon SH, Noh JH, Chung JM, Cho SI. Marked prevention of ischemic brain injury by Neu2000, an NMDA antagonist and antioxidant derived from aspirin and sulfasalazine. J Cereb Blood Flow Metab 2007;27:1142-51

Hawkins BT, Davis TP. The blood-brain barrier/neurovascular unit in health and disease. Pharmacol Rev 2005; 57:173-85

Hayashi Y, Jikihara I, Yagi T, Fukumura M, Ohashi Y, Ohta $\mathrm{Y}$, Takagi $\mathrm{H}$, Maeda M. Immunohistochemical investigation of caspase-1 and effect of caspase-1 inhibitor in delayed neuronal death after transient cerebral ischemia. Brain Res 2001;893:113-20

Hill JM, Switzer RC 3rd. The regional distribution and cellular localization of iron in the rat brain. Neuroscience 1984;11: 595-603

Hirase T, Staddon JM, Saitou M, Ando-Akatsuka Y, Itoh M, Furuse M, Fujimoto K, Tsukita S, Rubin LL. Occludin as a possible determinant of tight junction permeability in endothelial cells. J Cell Sci 1997;110:1603-13

Huang J, Upadhyay UM, Tamargo RJ. Inflammation in stroke and focal cerebral ischemia. Surg Neurol 2006;66:232-45

Jin G, Arai K, Murata Y, Wang S, Stins MF, Lo EH, van Leyen $\mathrm{K}$. Protecting against cerebrovascular injury: contributions of 12/15-lipoxygenase to edema formation after transient focal ischemia. Stroke 2008;39:2538-43

Kim GW, Lewen A, Copin J, Watson BD, Chan PH. The cytosolic antioxidant, copper/zinc superoxide dismutase, attenuates blood-brain barrier disruption and oxidative cellular injury after photothrombotic cortical ischemia in mice. Neuroscience 2001;105:1007-18

Kim KS. Mechanisms of microbial traversal of the blood-brain barrier. Nat Rev Microbiol 2008;6:625-34

Kitagawa K, Matsumoto M, Sobue K, Tagaya M, Okabe T, Niinobe M, Ohtsuki T, Handa N, Kimura K, Mikoshiba K, et al. The synapsin I brain distribution in ischemia. Neuroscience 1992;46:287-99

Kondo Y, Ogawa N, Asanuma M, Ota Z, Mori A. Regional differences in late-onset iron deposition, ferritin, transferrin, astrocyte proliferation, and microglial activation after transient forebrain ischemia in rat brain. $\mathrm{J}$ Cereb Blood Flow Metab 1995;15:216-26

Kuroiwa T, Shibutani M, Okeda R. Blood-brain barrier disruption and exacerbation of ischemic brain edema after restoration of blood flow in experimental focal cerebral ischemia. Acta Neuropathol 1988;76:62-70

Lum H, Roebuck KA. Oxidant stress and endothelial cell dysfunction. Am J Physiol Cell Physiol 2001;280:C719-41

Maier CM, Hsieh L, Crandall T, Narasimhan P, Chan PH. Evaluating therapeutic targets for reperfusion-related brain hemorrhage. Ann Neurol 2006;59:929-38

Matsuda R, Nishikawa A, Tanaka H. Visualization of dystrophic muscle fibers in $\mathrm{mdx}$ mouse by vital staining with Evans blue: evidence of apoptosis in dystrophin-deficient muscle. J Biochem 1995;118:959-64

Methy D, Bertrand N, Prigent-Tessier A, Mossiat C, Stanimirovic D, Beley A, Marie C. Beneficial effect of dipyridyl, a liposoluble iron chelator against focal cerebral ischemia: in vivo and in vitro evidence of protection of cerebral endothelial cells. Brain Res 2008;1193:136-42

Minagar A, Alexander JS. Blood-brain barrier disruption in multiple sclerosis. Mult Scler 2003;9:540-9

Park UJ, Lee YA, Won SM, Lee JH, Kang SH, Springer JE, Lee YB, Gwag BJ. Blood-derived iron mediates free radical production and neuronal death in the hippocampal CA1 area following transient forebrain ischemia in rat. Acta Neuropathol 2010. [Epub ahead of print]

Paul T. Effect of a prolonged superoxide flux on transferrin and ferritin. Arch Biochem Biophys 2000;382:253-61

Petty MA, Lo EH. Junctional complexes of the blood-brain barrier: permeability changes in neuroinflammation. Prog Neurobiol 2002;68:311-23

Preston E, Sutherland G, Finsten A. Three openings of the blood-brain barrier produced by forebrain ischemia in the rat. Neurosci Lett 1993;149:75-8

Pulsinelli WA, Buchan AM. The four-vessel occlusion rat model: method for complete occlusion of vertebral arteries and control of collateral circulation. Stroke 1988;19:913-4

Rezai-Zadeh K, Gate D, Town T. CNS infiltration of peripheral immune cells: D-Day for neurodegenerative disease? J Neuroimmune Pharmacol 2009;4:462-75

Sandoval KE, Witt KA. Blood-brain barrier tight junction permeability and ischemic stroke. Neurobiol Dis 2008;32: 
200-19

Sugawara T, Chan $\mathrm{PH}$. Reactive oxygen radicals and pathogenesis of neuronal death after cerebral ischemia. Antioxid Redox Signal 2003;5:597-607

Usatyuk PV, Parinandi NL, Natarajan V. Redox regulation of 4-hydroxy-2-nonenal-mediated endothelial barrier dysfunction by focal adhesion, adherens, and tight junction proteins. $J$ Biol Chem 2006;281:35554-66

Wang HK, Park UJ, Kim SY, Lee JH, Kim SU, Gwag BJ, Lee $Y B$. Free radical production in CA1 neurons induces MIP-1alpha expression, microglia recruitment, and delayed neuronal death after transient forebrain ischemia. J Neurosci
2008;28:1721-7

Won SJ, Kim DY, Gwag BJ. Cellular and molecular pathways of ischemic neuronal death. $\mathrm{J}$ Biochem Mol Biol 2002;35:67-86

Yin KJ, Deng Z, Hamblin M, Xiang Y, Huang H, Zhang J, Jiang $X$, Wang $Y$, Chen YE. Peroxisome proliferator-activated receptor delta regulation of miR-15a in ischemia-induced cerebral vascular endothelial injury. J Neurosci 2010;30: 6398-408

Zlokovic BV. The blood-brain barrier in health and chronic neurodegenerative disorders. Neuron 2008;57:178-201 